\title{
Effect of Costus igneus Leaf Juice on Antidiabetic Activity of Glimepiride in Rats - A Suggestive Study
}

\author{
K.A. Akhil1,*, Bheemachari² \\ ${ }^{1}$ Dr. HLT College of Pharmacy, Kengal, Channapatna - 562 161, Karnataka, India. \\ ${ }^{2}$ Department of Pharmacology, SAC College of Pharmacy, BG Nagar - 571 448, Karnataka, India.
}

\section{A R T I C L E DET A I LS}

Article history:

Received 30 March 2019

Accepted 20 April 2019

Available online 06 May 2019

\section{Keywords:}

Glimepiride

Costus igneus

Albino Rats

\begin{abstract}
A B S T R A C T
Due to therapeutic efficacy, Costus igneus Nak. (CI), (popularly known as insulin plant) is one medicinal plant in the limelight of having cure for diabetes. An oral diabetic agent Glimepiride was selected to evaluate the activity in per se and in combination with Costus igneus. The study drugs (glimepiride or Costus igneus leaf juice) were administered orally and blood glucose levels were determined using Glucometer at stipulated time intervals. It perhaps works in pathophysiologic/metabolic deranged conditions as observed by its per se effect in streptozotocin-induced diabetic rats' contrast to its effect in normal rats. The results of our study confirm that, the combined action of glimepiride and Costus igneus leaf juice has greater antidiabetic effects than their individual effects (per se).
\end{abstract}

\section{Introduction}

Diabetes mellitus (DM) is one such complex and multifarious group of disorders that disturbs the metabolism of carbohydrates, proteins and fats. It results either due to shortage or lack of insulin secretion or reduced sensitivity of the tissue to insulin [1]. The overall outcome is persistent hyperglycemia; which if uncontrolled leads to several secondary diseases/ disorders.

As per the World Health Organization (WHO), the global incidence of diabetes was 171 million in 2000 , and is projected to rise up to 366 million in 2030 [2]. The prevalence of diabetes in India is alarmingly high; World Health Organization (WHO) estimated that in 2010 there were 50.8 million people with diabetes in India and by 2030 number is expected to rise to 87 million.

The presence of DM confers increased risk of several devastating complications like cardio vascular disease and peripheral vascular diseases complications such as coronary artery disease, stroke, neuropathy, renal failure, retinopathy amputations and blindness. Persistent hyperglycemia/uncontrolled diabetes are attributed to be responsible for precipitation of majority of secondary diseases/ disorders in diabetic subjects. Hence tight glycemic regulation is very much essential for diabetic subjects to lead Quality of Life (QoL).

The current criteria for the diagnosis of DM emphasize that fasting plasma glucose (FPG) is the most reliable and convenient test for identifying DM in asymptomatic individuals. FPG $\geq 7.0 \mathrm{mmol}(126 \mathrm{mg} / \mathrm{dL})$ warrants the diagnosis of DM. A random plasma concentration $\geq 11.1$ mmol/L $(200 \mathrm{mg} / \mathrm{dL})$ accompanied by classical symptoms of DM (polyuria, polydipsia, and weight loss) is sufficient for the diagnosis of DM [3]. The impairment of glucose metabolism clearly starts when the fasting glucose concentrations exceed about $7.78 \mathrm{mmol} / \mathrm{L}(140 \mathrm{mg} / \mathrm{dL})$.

Management of DM includes optimum therapy, appropriate nutrition, and required level of exercise. Glycaemic control is central to optimum DM therapy; DM care includes detection and management of DM-specific complications and modification of risk factors for DM-associated diseases.

Insulin is the major therapeutic agent used for DM, particularly for type 1 DM. Parenteral therapy includes insulin, Glucagon like Peptide-1, and amylin. Oral glucose-lowering agents currently in use are biguanides, insulin secretagogues (sulfonylureas, repaglinide, nateglinide, etc.), thiazolidinediones, $\alpha$-glucosidase inhibitors, dipeptidyl peptidase-4 (DPP4) inhibitors, nutraceuticals and so on [5].

The major OHAs used in the treatment of type 2 DM are sulfonylureas, biguanides, repaglinide, nateglinide, and thiazolidinediones, agents that enhance GLP-1 receptor signaling, $\alpha$-glucosidase inhibitors, and inhibitors of sodium glucose cotransporter-2 (SGLT2).

Among the different OHAs clinically sulfonylureas are the most widely used drugs perhaps due to their well-established clinical safety and efficacy.

\subsection{Role of Medicinal Plants in Management of Diabetes Mellitus}

The use of medicinal plants in the treatment/management of the human ailments is of course as old as disease in itself. Many plant species have been used to treat life-threatening diseases including DM. A study conducted by World Health Organization (WHO) reveals that $80 \%$ of the world population solely relies on medicinal plants for their primary health care needs [6]. Costus igneus Nak. (CI), (popularly known as insulin plant) is one such medicinal plant in the limelight and is capable of having cure for diabetes. Leaf of this herbal plant helps to build up insulin by strengthening $\beta$-cells of pancreas in the human body, hence popularly known as "Insulin plant" in India [7]. This plant has large and ample leaves which are spirally disposed around the stem. In southern India, it usually grows as an ornamental plant and its leaves are used as a dietary supplement in the treatment of diabetes mellitus [8].

It is quite but natural tendency of human beings that, they will always be in the pursuit of alternative modalities / therapies which would make them to get rid of suffering and make them to lead a normal life as before. To fulfill their ambition the easy approaches are traditional or herbal drugs for which there is a wide propaganda through ads in both print and electronic media. Oral canvassing by the sufferers, particularly the senior citizens is the major media for wide use of several plant-based medicines in their different forms including consumption of raw leaves as well as leaf juice etc.

Consumption of fresh leaf juice of Costus igneus Nak. (CI) [Family: Costaceae] plant once daily for cure of DM/regulation of blood glucose levels by the diabetic subjects is in limelight these days. But neither the sufferers nor their advisers provide this information to their personal physicians/family members. But these diabetic subjects blood sugar levels have been possibly regulated using any one of the OHAs. Under such conditions there is every possibility of interference of juice on glycemic regulation achieved by $\mathrm{OHA}$ and may push the patient towards the lifethreatening situation. Considering this, in the present project an attempt has been made to evaluate the possible outcomes (both beneficial and harmful effects) of simultaneous use of insulin plant juice and oral antidiabetic drug glimepiride (widely used clinically) in both healthy and streptozotocin-induced diabetic rat models as a suggestive study. 


\section{Experimental Methods}

This descriptive study was conducted at SAC College of Pharmacy, B.G. Nagara, Karnataka.

\subsection{General Requirements}

Tween $80(1 \% \mathrm{w} / \mathrm{v})$ is used as vehicle for glimepiride administration throughout the study. The studied plant sample Costus igneus is collected from Kunigal area, Tumkur district, Karnataka in India. The plant sample Costus igneus was identified and authenticated by renowned botanist Prof. L.B. Kulkarni, Head, Department of Botany, Sri Prabhu Arts and Science College, Surpur, Karnataka.

Glimepiride used in the study is the generous compliments from M/s. Rakshith Pharmaceutical Limited, Vishakapatanam, AP. The same may be considered as their source of procurement throughout the study.

Streptozotocin : Purchased from Yarrow Chem. Products, Mumbai. Glimepiride : Rakshith Pharmaceutical Limited, Vishakapatanam, AP.

\subsection{Acute Toxicity Studies}

Several researchers have published data on acute toxicity profile of Costus igneus leaf and its different extracts. These study results reveal that, Costus igneus is nontoxic and safe upto the dose of $5000 \mathrm{mg} / \mathrm{kg}$ [9]. Based on published earlier research data and current practice by the diabetic patients in the society fresh juice of Costus igneus leaf fresh juice is used in the project to simulate the real scenario.

Glimepiride is used orally in clinical practice; hence same route is followed. Glimepiride is poorly water soluble, hence to have proper administration glimepiride pure drug was suspended in Tween 80 $(1 \% \mathrm{w} / \mathrm{v})$ and used [10]. The suspension of drug was freshly prepared in such a way to yield the required dose of the drug in maximum allowed volume of the vehicle for rats. Costus igneus one fresh leaf is collected and its juice is prepared by grinding. This juice is consumed by the diabetic patients once in the morning [11]. To simulate the real situation in this study, fresh leaf juice of $C$. igneus is used in the study. The Costus igneus leaf was triturated and pressed in mortar and the juice is collected for administration to rats.

\subsubsection{Study Design}

The study was carried out in normal and streptozotocin-induced diabetic rats to evaluate the per se and combined effects. The following scheme of study (Table 1) was used for both normal and diabetic rats.

Table 1 General scheme of the study

\begin{tabular}{lll}
\hline Stage-1 & Stage-2 & Stage-3 \\
\hline $\begin{array}{l}\text { Per Se effect of Tween } \\
80(1 \% \mathrm{w} / \mathrm{v})\end{array}$ & $\begin{array}{l}\text { Per Se effect of } \\
\text { Glimepiride }(10 \mathrm{mg} / \mathrm{kg} \\
\text { body weight })\end{array}$ & $\begin{array}{l}\text { Combined effect of } \\
\text { Glimepiride \& Costus igneus } \\
\text { leaf juice }(0.5 \mathrm{~mL})\end{array}$ \\
\hline
\end{tabular}

All the stages are repeated in streptozotocin-induced diabetic rats. On the days of glimepiride administration, additional glucose solution for oral administration was made available in order to take care of any severe hypoglycemia so as to prevent the death of the study animals.

\subsubsection{Estimation of Blood Glucose}

Accu Check Active Glucometer is used for the current study. The apparatus is reported to give convenient and accurate results [12]. Works on systems based on dry-reagent test strips with visually read end-points and/or simple-to-use reflectance meters and biosensors (minimized operator-dependent steps).

The blood drops from the caudal vein are fed to the strips. The system based dry reagent strips interact with the blood and provide the digital results based on bio-sensor mechanism.

The studies were carried out in the Department of Pharmacology at SAC College of Pharmacy, B G Nagara, Karnataka, which is duly licensed by the CPCSEA (Committee for the Purpose of Control and Supervision of Experiments in Animals; 377/CPCSEA Dated 22/02/2001). The study protocols were approved according to current regulations of CPCSEA by the Institution Animal Ethics Committee for studies on rats.

\subsubsection{Animals}

The rats used in the study were taken from the Animal house of Sri. Adichunchanagiri College of Pharmacy, B.G. Nagara (377/CPCSEA Dated 22/02/2001) and were housed under standard husbandry conditions. Hence the same may be considered as source of animal procurement in the subsequent sections. Rats (either sex) were selected for the current study.

\subsubsection{Method for Oral Administration}

A 16 gauge needle whose sharp tip was gently trimmed, taking due care not to collapse its bore so as to yield smooth open edge. Over the entire length of the stainless-steel part of the needle, suitable flexible polythene tubing was slipped so as to project a very small length after the needle tip. This extra projected tube by virtue of its softness prevents the possibility of damage in the oral cavity while administration. The terminal part of the needle was shaped to blunt to enable convenient and perfect administration. The needle so modified was fixed to $1 \mathrm{ml}$ tuberculin syringe for administration. For intra gastric administration, the rat was grasped firmly in left hand and the modified needle was inserted right into the oesophagus and the plunger was gently pushed. This act was followed by administration of $0.2 \mathrm{~mL}$ of distilled water to ensure correct dose administration.

\subsubsection{Method for Blood Sampling}

The rat was placed in a rat restrainer. The tail was pulled out and the tip of tail was thin sliced $(0.05 \mathrm{~mm})$ using sharp scissors. The blood drops (each sample $0.05 \mathrm{~mL}$ ) were fed directly to strips with glucometer and reading was noted. Later dry cotton was applied for few minutes to stop further bleeding and the tail tip was sterilized by applying alcohol.

\subsubsection{Parameters Considered for Assessing Hypoglycemia}

These parameters were selected empirically by observation and earlier experience.

1. Onset of hypoglycemia: Time taken to reduce blood glucose levels to the extent of $20 \%$

2. Peak hypoglycemia: The maximum percentage of blood glucose reduction

3. Duration of hypoglycemia: The time duration in which minimum of $20 \%$ blood glucose reduction is observed.

Percentage reduction in blood glucose levels at any given time ' $t$ ' may be calculated with reference to the basal glucose level, using the following Eq.(1):

$$
\% \text { Blood glucose reduction at time ' } \mathrm{t}=\mathrm{A}-\mathrm{B} / \mathrm{A} \times 100
$$

where, $\mathrm{A}=$ Initial blood glucose level before vehicle/drug administration, $\mathrm{B}=$ Blood glucose levels at time ' $\mathrm{t}$ ' after the vehicle/drug administration.

The same procedure will be repeated in streptozotocin induced diabetic rats to understand the influence of vehicle on the blood glucose levels.

\subsubsection{Streprozotocin Induced Diabetes}

Adult ( 9 weeks old) male Albino rats were made diabetic with an intraperitoneal injection of streptozotocin (STZ, $65 \mathrm{mg} / \mathrm{kg}$ body weight) dissolved in citrate buffer $(0.1 \mathrm{M}, \mathrm{pH} 4.5)$. Streptozotocin injected animals exhibited massive glycosuria and hyperglycaemia within few days. Diabetes was confirmed in STZ rats by measuring the fasting blood glucose concentration, 96 hours after the injection with STZ. Albino rats with blood glucose level above $250 \mathrm{mg} / \mathrm{dL}$ were considered to be diabetic [13] and were used in this project. Six rats were injected with saline that served as control. Percentage reduction in blood glucose levels at any given time ' $t$ ' may be calculated with reference to the basal glucose level, using Eq.(1).

\subsection{Statistical Analysis}

The study results were analyzed using Graph Pad Prism software and expressed as mean \pm SEM $(n=6)$. Statistical analysis was performed using ANOVA followed by Dunnet's multiple comparison test. The results were considered statistically significant, if $\mathrm{p}$ value is $<0.05$.

\section{Results and Discussion}

Glimepiride is the most frequently prescribed drug among the other OHAs. Since it has proven history of safety, efficacy and once a day dose in contrast other sulfonylureas [14]. Hence instances of simultaneous use of glimepiride and Costus igneus juice by the diabetic subjects is more compared to other OHAs. Hence in the present project among the OHAs glimepiride is selected to understand the influence Costus igneus leaf juice on glycemic regulation achieved by using it in both normal and streptozotocin-induced diabetic rats.

The studies were conducted using albino rats for following reasons. As per the regulatory norms to study or understand the effect or mechanism of action of any drug (synthetic/herbal) it should be essentially carried out in animals only, preferably rats/mice. Second, most of the drug related 
complex mechanisms are well understood and established using animal models like rats. Last, conducting study using small animals like rats is most convenient, comparatively economical and results generated are acceptable and extrapolatable [15].

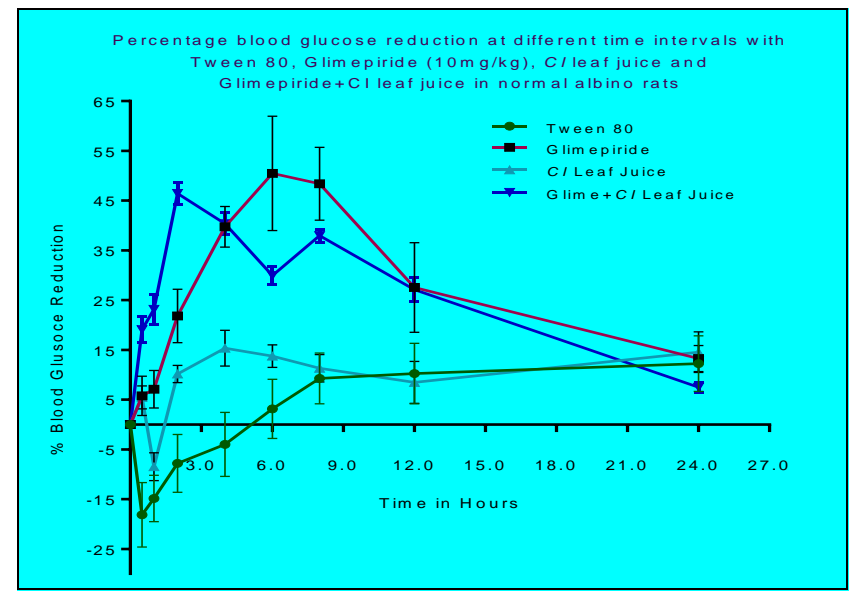

Fig. 1 Percentage reduction in blood glucose levels with Tween 80, Glimepiride, Costus igneus leaf juice and their combination in normal albino rats

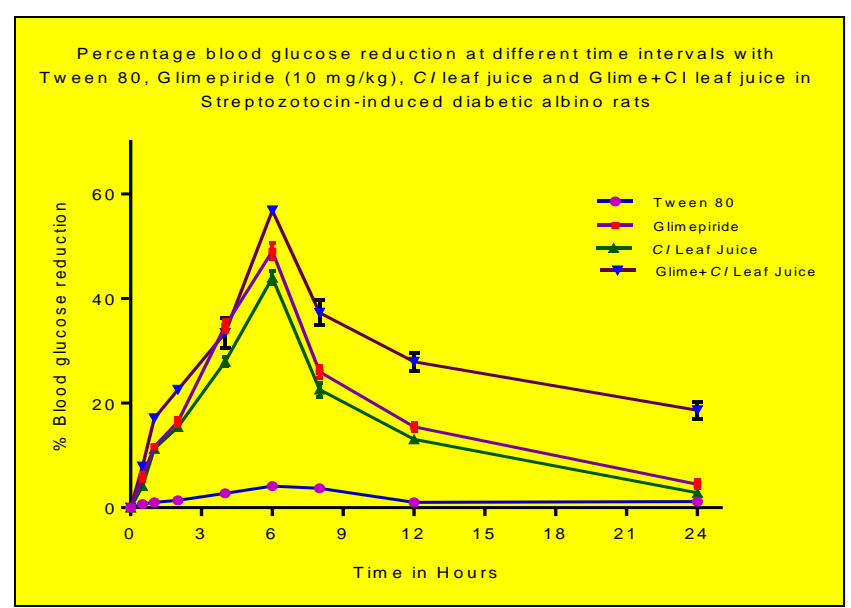

Fig. 2 Percentage reduction in blood glucose levels with Tween 80, Glimepiride, Costus igneus leaf juice and their combination in Streptozotocin induced diabetic albino rats

The first phase studies were conducted using normal rats; since to study and understand the effect of a drug/herb or influence of one agent (drug or herb) on the other, normal animas are ideal and preferable. Since in normal animals, the built in positive and negative feedback mechanisms and other body's homeostatic mechanisms are actively functional, as a result the observed/estimated results are more reliable [16].

The second phase studies were conducted in streptozotocin-induced diabetic rats. Evidence suggests that certain drugs/molecules show their effects/influence only in altered physiology or metabolic derangements [17]. Hence to understand and confirm specific activity (if any) the present study is conducted in streptozotocin-induced diabetic rats. Streptozotocin was used to induce the diabetes in rats [18]. Since in streptozotocininduced rats all pathophysiologic parameters and metabolic derangements are similar to those observed in patients with type $2 \mathrm{DM}$ [19].

Tween-80 per se administration did not produce any observable change in the blood glucose levels in healthy albino rats. This rule out the possibility of influence of tween 80 on blood glucose levels, which is used as vehicle for administration of glimepiride in rats.

Glimepiride per se administration exhibited its onset of action at $2^{\text {nd }}$ hour of its administration by reducing the blood glucose levels by $21.81 \%$. It exhibited its peak hypoglycemic effect at $6^{\text {th }}$ hour by reducing $50 \%$ of blood glucose compared to its basal level (observed at " 0 " hour). The duration of hypoglycemic effect was observed for 10 hours (from $2^{\text {nd }}$ hour of its administration to $12^{\text {th }}$ hour). Costus igneus leaf juice administration did not produce any significant hypoglycemic effect in normal rats. The maximum ability of Costus igneus leaf juice was to reduce the blood glucose levels by $15.34 \%$ at $4^{\text {th }}$ hour of its administration.

Costus igneus leaf juice is believed to reduce the blood glucose levels in diabetic patients. However, in normal rats it did not produce any significant reduction in blood glucose levels. Hence there may be possibility that the herb may produce effect in metabolic derangement or pathophysiologic condition like DM.

Glimepiride and Costus igneus leaf juice when they are simultaneously administered in normal rats, surprisingly the hypoglycemic ability of glimepiride was attenuated. The hypoglycemic action begun at 0.5 hours with reduction of blood glucose by $19.07 \%$. the peak hypoglycemic effect of combination was observed at $2^{\text {nd }}$ hour with reduction of blood glucose levels by $46.45 \%(\mathrm{p}<0.05)$. The duration of hypoglycemic action was seen upto 12 hours.

Costus igneus leaf juice co-administration with glimepiride altered both the pharmacokinetic and pharmacodynamics profiles of the combination. With regard to glimepiride the onset of hypoglycemic action is preponed from $2^{\text {nd }}$ hour when administered alone to 0.5 hours when administered along with Costus igneus leaf juice. The peak hypoglycemic effect is also preponed from $6^{\text {th }}$ hour to $2^{\text {nd }}$ hour. However, the intensity of hypoglycemic effect is also reduced from $50.47 \%$ to $46.45 \%$ when simultaneously administered with Costus igneus leaf juice. The duration of hypoglycemic effect of combination is $\mathbf{1 1 . 5}$ hours in contrast to 10 hours observed with glimepiride alone.

The pharmacokinetic and pharmacodynamics changes observed following simultaneous administration of glimepiride and Costus igneus leaf juice could be due to facilitation of absorption, distribution and excretion of glimepiride by the juice as evident from the observations. From this it is also evident that, Costus igneus leaf juice has not contributed on its own in reduction of blood glucose levels in normal rats even when used along with glimepiride. Hence Costus igneus leaf juice is devoid of any significant hypoglycemic action in normal animals and is of low hypoglycemic risk (Fig. 1).

However, the results observed with streptozotocin-induced diabetic rats are quite interesting and surprising. The per se administration of tween 80 did not produce any significant antidiabetic effect, which rules out the influence of vehicle on antidiabetic potency of glimepiride. The per se administration of glimepiride exhibited late onset of action observed around $2^{\text {nd }}$ hour and duration of action upto $12^{\text {th }}$ hours. The peak antidiabetic action was observed at $6^{\text {th }}$ hour and there was reduction of blood glucose level by $49.02 \%(\mathrm{p}<0.05)$ which is almost similar with what is observed in normal rats.

The per se administration of Costus igneus leaf juice indeed produced surprising and quite interesting results as evident from the results. The antidiabetic action started between $2^{\text {nd }}$ and $4^{\text {th }}$ hours of its administration. The juice exhibited peak antidiabetic effect at $6^{\text {th }}$ hour by reducing the blood glucose levels by $43.97 \%$ which indeed comparable with glimepiride a synthetic drug (49.02\%). The duration of antidiabetic action is also up to $8^{\text {th }}$ hour of its administration (6 hour approximately).

Certain drugs like metformin and paracetamol are known to act only in metabolic derangements [20]. Perhaps the juice of Costus igneus leaf works in similar fashion like above drugs. Since in normal rats the juice did not produce any significant hypoglycemic effect. However, in streptozotocin-induced diabetic rats (metabolic derangement/pathophysiologic condition) it has produced wonderful results. Costus igneus leaf juice per se administration would be sufficient to keep glycemic regulation in borderline diabetics. This effect of Costus igneus leaf juice is due to its claimed insulin-like action as reported by earlier researchers [21]. The juice might have dose related antidiabetic action, hence regulated volume of juice either in terms of number of leaves to be used or total volume of juice to be must be well determined before it is consumed with other synthetic OHAs.

When glimepiride and Costus igneus leaf juice were simultaneously administered to streptozotocin-induced diabetic rats, it produced interesting results. The results depicts that, the onset of antidiabetic action was observed between $1^{\text {st }}$ and $2^{\text {nd }}$ hour of their administration. The peak antidiabetic effect was observed at $6^{\text {th }}$ hour with reduction of blood glucose levels by $56.81 \%$ contrast to $46.45 \%$ observed in normal rats $(p<0.05)$. Further the antidiabetic action was extended much beyond 12 hours and closer to 24 hours (Fig. 2).

From all the above it is quite clear and evident that, the Costus igneus leaf juice has insulin-like action. It perhaps works in pathophysiologic/metabolic deranged conditions as observed by its per se effect in diabetic rats contrast to its effect in normal rats.

The results of the study and its underlying mechanisms as discussed in the above sections confirm that, the combined action of glimepiride and Costus igneus leaf juice has greater antidiabetic effects than their individual effects (per se). Hence exercise of care is essential with regard to either the dose of glimepiride or volume of the Costus igneus leaf juice when they are simultaneously used to avoid hypoglycemia and related clinical emergency instances.

Further our study observations show that, $C I$ leaf juice produces its effect in metabolic derangements or pathophysiologic conditions like DM. 
Thus, the literature reveals that, the antidiabetic activity of $C I$ plant is attributed to the presence of insulin like protein (ILP), quercetin, polyphenols, flavonoids, terpenoids, coumarins and other constituents which show reduction in blood glucose levels.

The antidiabetic effect can be due to release of insulin, insulinsensitizing action or a combination of both. However, further studies need to be undertaken to understand the mechanism of action by measurement of either insulin or ' $\mathrm{C}$ ' peptide level.

\section{Conclusion}

This study resolutely proven the efficacy of simultaneous administration of glimepiride and Costus igneus leaf juice to streptozotocin-induced rats produced significant antidiabetic effect greater than the individual effects exhibited by either glimepiride or Costus igneus leaf juice on their own. Further our study observations propose that, Costus igneus leaf juice produces its effect perhaps only in metabolic derangements or pathophysiologic conditions like DM. From our study it is concluded that, when OHAs like glimepiride is used along with Costus igneus leaf juice by the diabetic subjects, there are chances of dose related hypoglycemia and associated risks. Hence restructuring of dose of either glimepiride or quantity of juice is necessary to avoid the untoward effects. Our study has opened an avenue to the researchers to explore the detailed scientific basis of bizarre type of behavior of Costus igneus leaf juice both in the presence and absence of glimepiride and also in normal as well as pathophysiologic conditions. This study highlights the potential antidiabetic activity for Costus igneus, suggesting that, with further research and refinement, Costus igneus leaf extracts could find a place in the treatment of Diabetes Mellitus. Further in vitro and in vivo studies on a large number of clinical isolates are necessary to further investigate and standardize the effect of Costus igneus. Neverthe- less, the promising results in this study may open a window for potential new antidiabetics.

\section{Acknowledgement}

The authors thank the faculty and management of Sri Adichunchanagiri College of Pharmacy, BG Nagara, Karnataka and Rajiv Gandhi University of Health Sciences, Bangalore for constant support and encouragement.

\section{References}

[1] S.K. Prasad, Alka Kulshreshtha, Taj N. Qureshi, Antidiabetic activity of some herbal plants in streptozotocin induced diabetic albino rats, Pakistan J. Nutr. 8(5) (2009)551-557.
[2] N. Kanivalan, R. Rajakumar, P. Mani, Anti-diabetic and hypolipidamic effects of costus igneus leaves extracts against streptozotocin induced diabetic albino rats, Acta Biomed. Sci. 1(2) (2014)74-79.

[3] W. Kerner, J. Brückel, Definition, classification and diagnosis of diabetes mellitus, German Diabetes Associaton, Clin. Prac. Guide.122 (2014) 384-386.

[4] Silke Crommen, Marie-Christine Simon, Microbial regulation of glucose metabolism and insulin resistance, Genes 9(10) (2018)1-4.

[5] Andrew J. Krentz, Clifford J. Bailey, Oral antidiabetic agent's current role in Type 2 diabetes mellitus, Drugs 65(3) (2005) 385-411.

[6] S. Elavarasi, K. Saravanan, Ethnobotanical study of plants used to treat diabetes by tribal people of Kolli hills, Namakkal District, Tamilnadu, Southern India, Int. J. Pharmtech Res. 4(1) (2012) 404-411.

[7] Saravanan, S. Karunakaran, P. Vivek, S. Dhanasekaran, Studies on antibacterial activity of root extract of Costus igneus, Int. J. Chemtech Res. 6(9) (2014) 42014206.

[8] Ravindra B. Malabadi, Raju K. Chalannavar, Neelambika T. Meti, Ramesh S Gani, Sudabattula Vijayakumar, et al., Insulin plant, costus specious: ethnobotany and pharmacological updates, Int. J. Curr. Res. Biosci. Plant Biol. 3(7) (2016) 151-161.

[9] Bhat Vishnu, Asuti Naveen, Kamat Akshay, Sikarwar Mukesh, M.B. Patil, Antidiabetic activity of insulin plant (Costus igneus) leaf extract in diabetic rats, Jour. Pharm. Res. 3(3) (2010) 608-611.

[10] Marwa M.A. Khalaf, Gamal A. El Sherbiny, Hekma A. AbdEllatif, Afaf A. Ain shoka, Mostafa E. El Sayed, et.al, Comparative effects of glimepiride, vanady sulfate and their combination on hypoglycemic parameters and oxidative stress, Brit. J. Pharmacol. Toxicol. 3(6) (2012) 278-288.

[11] Shetty Aj, S.M. Parampalli, R. Bhandarkar, S. Kotian, Effect of the insulin plant (Costus igneus) leaves on blood glucose levels in diabetic patients: A cross sectional study, J. Clin. Diagnostic Res. 4 (2010) 2617-2621.

[12] Guido Freckmann, Annette Baumstark, Nina Jendrike, Eva Zschornack, Serge Kocher, et.al, System accuracy evaluation of 27 blood glucose monitoring systems according to Din En ISO 15197N, Diabetes Technol. Therapeutics 12(3) (2013) 221-231.

[13] WHO, Report of a WHO Consultation, Definition, diagnosis and classification of diabetes mellitus and its complications. WHO, Geneva, 1999.

[14] Draft Guidance, Draft Guidance on approval of clinical trials \& new drugs, Central Drugs Standard Control Organization Directorate General of Health Services Ministry of Health \& Family Welfare, Govt. of India, India, 2011.

[15] K. Srinivasan, P. Ramarao, Animal models in type 2 diabetes research: An overview, Ind. J. Med. Res. 125 (2007) 451-472.

[16] Peter Singer, Animal liberation, Ind. J. Med. Res. 125 (2007) 306-325

[17] Rafik Karaman, Commonly used drugs- uses, side effects, bioavailability and approaches to improve it, Nova Science Publishers, Inc., NY, USA, 2008.

18] Akbarzadeh, D. Norouzian, M.R. Mehrabi, Sh. Jamshidi, A. Farhangi, et al, Induction of diabetes by streptozotocin in rats, Ind. J. Clin. Biochem. 22(2) (2007) 60-64.

[19] Campbell, M. Jared, Bellman, M. Susan, Stephenson, et al., Metformin reduces all-cause mortality and diseases of ageing independent of its effect on diabetes control: a systematic review and analysis, Ageing Res. Rev. 40 (2017) 31-34.

[20] Morta Jozwiak, Jerry Niwak, Paracetamol mechanisms of action, applications and safety concern, Acta Poloniae Pharma. Drug Res. 71(1) (2014)11-23.

21] C. Bindu, P. Rama Bhat, G. Krishna Prasad, B.C. Girish, Relative potency of antidiabetic effect of costus speciosus (Koen. Ex Retz.) smith plant extract in mouse fibroblast cell line, Int. J. Res.- Granthaalayah 4(3) (2016) 99-106. 